\title{
Effect of cigarette smoke extract on mitochondrial division in mouse quadriceps femoris cells
}

\author{
Zhidan Tan ${ }^{1}$, Siqi $\mathrm{Li}^{1}$, Su Zhu ${ }^{2}$, Xiaoxuan $\mathrm{Yao}^{2}, \mathrm{Jing}^{3}$, Xinglin Gao ${ }^{3 \#}$, Shifang Yang ${ }^{3 \#}$ \\ ${ }^{1}$ The Second School of Clinical Medicine, Southern Medical University, Guangdong Provincial People's Hospital, Guangdong Academy of Medical \\ Sciences, Guangzhou, China; ${ }^{2}$ Shantou University Medical College, Shantou, China; ${ }^{3}$ Department of Respiratory and Critical Care Medicine, \\ Guangdong Provincial People's Hospital, Guangdong Academy of Medical Sciences, Guangdong Provincial Geriatrics Institute, The Second School \\ of Clinical Medicine, Southern Medical University, Guangzhou, China \\ Contributions: (I) Conception and design: X Gao, S Yang; (II) Administrative support: X Gao, S Yang; (III) Provision of study materials or patients: J \\ Li; (IV) Collection and assembly of data: Z Tan, S Li, S Zhu, X Yao; (V) Data analysis and interpretation: Z Tan, S Li, S Zhu, X Yao; (VI) Manuscript \\ writing: All authors; (VII) Final approval of manuscript: All authors. \\ "These authors contributed equally to this work. \\ Correspondence to: Shifang Yang; Xinglin Gao. Department of Respiratory and Critical Care Medicine, Guangdong Provincial People's Hospital, \\ Guangdong Academy of Medical Sciences, Guangdong Provincial Geriatrics Institute, The Second School of Clinical Medicine, Southern Medical \\ University, Guangzhou 510515, China. Email: yangshifang@gdph.org.cn; xinglingao@hotmail.com.
}

Background: To observe the effect of cigarette smoke extract (CSE) on mitochondrial division in mouse quadriceps femoris cells and to explore the potential molecular mechanism of skeletal muscle dysfunction (SMD) in patients with chronic obstructive pulmonary disease (COPD).

Methods: Quadriceps femoris were cultured, passaged, and stimulated with different concentrations of CSE. We divided cells into four groups (Control, 2.5\%, 5\%, 10\%). The growth of cells, the expression of Dynamin related protein 1 (Drp-1), and apoptosis were observed and evaluated by fluorescence microscopy, RT-PCR, Western blot, and flow cytometry.

Results: The longer the intervention time, the more obvious the decrease in cell number. In the $5 \%$ and $10 \%$ groups, the cells became round with gaps. Under an inverted fluorescence microscope, the green fluorescence of cells in 5\% and 10\% stained with Mito-Tracker Green was significantly less than that of the Control and $2.5 \%$. Red fluorescence was reduced and the green fluorescence was increased in the $5 \%$ and $10 \%$ stained with JC-1. Flow cytometry analysis showed that reactive oxygen species (ROS) and apoptosis were increased in the CSE intervention groups. In the Control, $2.5 \%, 5 \%$, and $10 \%$, the levels of ROS were $0.052 \pm 0.015,0.170 \pm 0.030,5.340 \pm 0.500$, and $24.400 \pm 1.900$, respectively. The apoptotic rates (\%) were $0.270 \pm 0.009,2.650 \pm 0.060,11.850 \pm 0.020$, and $31.820 \pm 1.260$, respectively. The relative expression levels were, $0.900 \pm 0.093,1.141 \pm 0.099,1.361 \pm 0.034,2.155 \pm 0.092$ for DNM1L mRNA, and $0.509 \pm 0.008,0.569 \pm 0.028$, $0.792 \pm 0.048,0.940 \pm 0.062$ for Drp-1. There were significant differences in the apoptotic rate, and Drp-1 expression between $5 \%$ and $10 \%$ compared with the Control and $2.5 \%(\mathrm{P}<0.05)$.

Conclusions: CSE may enhance mitochondrial division of quadriceps femoris cells by up-regulating the expression of Drp-1, affecting cellular energy metabolism and promoting quadriceps femoris apoptosis, ultimately leading to the occurrence and development of skeletal muscle dysfunction in COPD.

Keywords: Chronic obstructive pulmonary disease (COPD); quadriceps femoris; Dynamin related protein 1 (Drp-1); cigarette smoke extract (CSE)

Submitted Oct 15, 2021. Accepted for publication Nov 19, 2021.

doi: $10.21037 / \mathrm{atm}-21-5891$

View this article at: https://dx.doi.org/10.21037/atm-21-5891 


\section{Introduction}

Chronic obstructive pulmonary disease (COPD), characterized by persistent respiratory symptoms and airflow limitation, is a systemic disease that not only affects the lungs, but also leads to skeletal muscle dysfunction (SMD) and other system dysfunction (e.g., cardiovascular system, nervous system, and digestive system, among others). The major comorbidities may affect morbidity and mortality. In recent years, more and more studies have found that in COPD patients with exercise limitation, about $40 \%$ of patients do not have severely impaired pulmonary function, but present with significant SMD (1). The occurrence of SMD in COPD patients is a complex mechanism involving multiple factors and multiple molecular biological mechanisms, which is not yet fully understood. Mitochondria are the cellular energy machinery. The balance between their division and fusion is the basis for the normal physiological functions of tissue cells. Dynamin related protein 1 (Drp-1) is a key protein which initiates the division of mitochondria and is mainly located in the cell matrix, mediating the division process of mitochondria $(2,3)$. At present, fewer studies have focused on the effect of cigarette smoke extract (CSE) on mitochondria, while we want to find out the association between the two in COPD. Therefore, we propose that CSE increases Drp-1 expression, leading to increased mitochondrial division and accelerating apoptosis of cells, which leads to SMD in COPD. In our study, mouse quadriceps myocytes were used to observe the effects of different concentrations of CSE on mitochondria, Drp-1 expression, and apoptosis.

We present the following article in accordance with the MDAR reporting checklist (available at https://dx.doi. org/10.21037/atm-21-5891).

\section{Methods}

\section{Source of cells}

The mouse myoblast cell line $\mathrm{C} 2 \mathrm{C} 12$ was purchased from the Shanghai Institute of Cell Biology, Chinese Academy of Sciences and cultured with DMEM high-glucose medium containing $10 \%$ fetal bovine serum (FBS). Cells in logarithmic growth phase were obtained for subsequent experiments at passage 5 .

\section{Main reagents and instruments}

DMEM high-glucose medium and FBS were purchased from Gibco. The Mito-Tracker Green kit and JC-1 kit were purchased from Beyotime. The total RNA extraction reagent Trizol was purchased from Life Technologies. The rabbit anti-monoclonal antibody and HRP-labeled mouse anti-rabbit IgG were purchased from CST. The ROS assay kit was purchased from Beyotime, the apoptosis detection kit was purchased from $\mathrm{BD}$, the reverse transcription kit and qPCR kit were purchased from Accurate Biology, and primers were synthesized by Accurate Biology according to the designs detailed in Table 1.

\section{Study methods}

\section{Cell culture and intervention}

Mouse C2C12 myoblasts were cultured in DMEM highglucose medium containing $10 \% \mathrm{FBS}$ and passaged until passage 5. Cells in logarithmic growth phase were obtained and seeded in a 6 -well plate after counting with a cell counter. Cigarette smoke was extracted according to a previously described method (4) and filtered with a $0.22 \mu \mathrm{m}$ filter. Prepare the culture medium with DMEM, FBS and CSE. Set a CSE concentration gradient as $2.5 \%, 5 \%, 7.5 \%$, $10 \%, 12.5 \%, 15 \%$, etc. Microscopically, at concentrations greater than $10 \%$, cells float and die in large numbers within 6 hours. Therefore, to complete the experiment, CSE concentrations of $2.5 \%, 5 \%, 10 \%$ were used. They were divided into four groups, Control, $2.5 \%, 5 \%$, and $10 \%$. All of the following assays were performed with cells collected after $24 \mathrm{~h}$ of intervention.

\section{Fluorescence staining and microscopy}

Cells in 6-well plates were stained with Mito-Tracker Green and JC-1 according to the manufacturer's instructions. Cells were then observed and photographed under an inverted fluorescence microscope in the dark. The images were processed with Image $\mathrm{J}$ software to calculate the mean fluorescence values or ratios of different groups.

\section{Western blot}

RIPA/PMSF lysates were prepared at a ratio of 100:1 from cells treated with different concentrations of CSE for $24 \mathrm{~h}$ in a 6-well plate. Samples were lysed on ice for $30 \mathrm{~min}$, during which they were shaken 3 times, and cell lysates were then collected in EP tubes. After centrifugation at $12,000 \mathrm{rpm}$ for $20 \mathrm{~min}$ at $4{ }^{\circ} \mathrm{C}$, the supernatants were obtained for protein concentration determination by the BCA assay. A 10\% separation gel and 5\% stacking gel were prepared $30 \mu \mathrm{g}$ and $20 \mu \mathrm{L}$ denatured protein, 
Table 1 The sequences of the primers for real-time PCR

\begin{tabular}{lcc}
\hline Name & Primer sequence $\left(5^{\prime}-3^{\prime}\right)$ & Product (bp) \\
\hline Dnm1l & F: CAACAGGCAACTGGAGAGGAATG & 145 \\
& R: GCAACTGGAACTGGCACATCT & \\
GAPDH & F: GGCCTCCAAGGAGTAAGACC & 122 \\
& R: AGGGGAGATTCAGTGTGGTG & \\
\hline
\end{tabular}

and electrophoresis was performed at $80 \mathrm{mV}$ for $30 \mathrm{~min}$, then voltage was increased to $120 \mathrm{mV}$. When the marker indicator band dropped to the stacking gel, electrophoresis was continued for $120 \mathrm{~min}$, then the proteins were transferred to a PVDF membrane after the indicator band reached the bottom of the gel. Membranes were blocked on a shaker at room temperature for $1 \mathrm{~h}$ in $5 \%$ skimmed milk powder prepared with fresh TBST. Primary antibodies for Drp-1 (rabbit mAb) and GAPDH were added and incubated overnight at $4{ }^{\circ} \mathrm{C}$. Membranes were washed 3 times with TBST for 10 min each time, and the secondary antibody HRP-labeled mouse anti-rabbit IgG was added and incubated for $2 \mathrm{~h}$. After washing again 3 times with TBST for $10 \mathrm{~min}$ each time at $4^{\circ} \mathrm{C}$, chemiluminescence was used for development. Image J software was used to analyze the gray values of each band. The relative gray values were calculated with the internal reference gray level.

\section{RT-PCR}

Cells with CSE intervention for $24 \mathrm{~h}$ were collected, total RNA was extracted with Trizol, and cDNA was synthesized by reverse transcription according to the reverse transcription kit instructions. Primer sequences were designed and synthesized by Accurate Biology using Primer5 software. Detection was performed with a BIORAD fluorescence quantitative PCR instrument. A $10 \mu \mathrm{L}$ reaction system was prepared according to the requirements of the instructions and 3 auxiliary wells were set for each sample. The samples were loaded separately and the test was then carried out on the machine. The reaction procedure settings for the two-step method were as follows: $95^{\circ} \mathrm{C}$ for $30 \mathrm{~s} ; 95^{\circ} \mathrm{C}$ for $5 \mathrm{~s} ; 60^{\circ} \mathrm{C}$ for $30 \mathrm{~s}$, for 40 cycles in total, and the dissolution curve was added.

\section{Flow cytometry}

An ROS assay kit was used to detect cellular ROS changes after CSE intervention. The cell supernatants in 6-well plates were collected and the cells were washed twice with pre-chilled PBS. The DCFH-DA probe was diluted according to the instructions, an appropriate amount of probe was added to each well, and a positive control group was also set up. After incubation in a cell incubator for $20 \mathrm{~min}$, the cells were collected after digestion with trypsin without EDTA. Cells were resuspended twice with PBS after centrifugation and detected in the dark. FlowJo 10.4 was used to analyze the experimental results.

The Annexin V-PE double staining method was used to detect apoptosis after CSE intervention. The cell supernatants in 6-well plates were collected and the cells were washed twice with pre-cooled PBS. The cells were collected after trypsin digestion without EDTA. The cells were resuspended twice with PBS and centrifuged at $1,000 \mathrm{rpm}$ for $5 \mathrm{~min}$, and then the supernatant was removed. The cell concentration was adjusted to $1 \times 10^{6} / \mathrm{L}$ with $1 \times$ binding buffer, and $5 \mu \mathrm{L}$ Annexin V-FITC and $5 \mu \mathrm{L}$ PI were added to $100 \mu \mathrm{L}$ of cells. The cells were gently mixed and incubated at room temperature for $15 \mathrm{~min}$ in the dark, and $400 \mu \mathrm{L}$ of $1 \times$ binding buffer was added. At the same time, a blank tube and Annexin V-FITC and PI monochrome tubes were set and detected on the machine in the dark. The experimental results were analyzed using FlowJo 10.4.

\section{Statistical analysis}

The simultaneous duplication of the same generation of cells was defined as technical duplication, while the duplication of different generations of cells cultured at different times was defined as biological duplication. For each experiment, we have three technical and biological replicates. All the experimental results were used for statistical purposes. Analysis was performed with SPSS 20.0 statistical software. Data were expressed as mean \pm standard deviation (mean $\pm \mathrm{SD}$ ). One-way ANOVA was used for comparison between multiple groups, and the SNK-q test was used for pairwise comparison. $\mathrm{P}<0.05$ indicated a statistically significant difference.

\section{Results}

\section{Cell morphological and mitochondria changes after CSE intervention}

Under bright field observation with an inverted microscope, control cells were found to extended outwards in a spindle shape, fused with each other, and were arranged regularly in a certain direction. However, with the increase of 


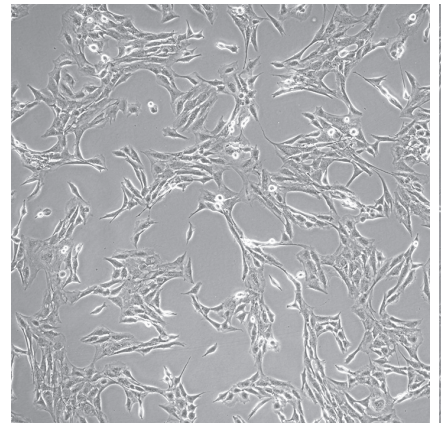

Control

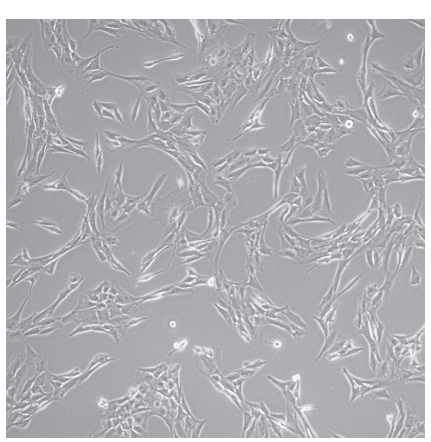

$2.5 \%$

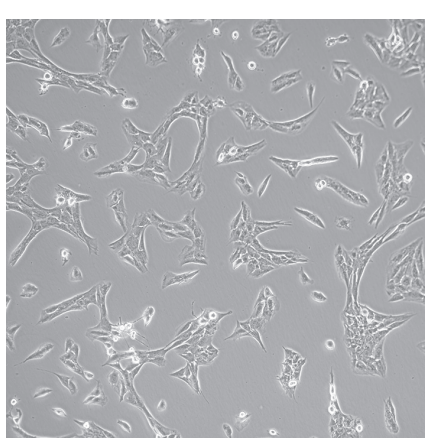

$5 \%$

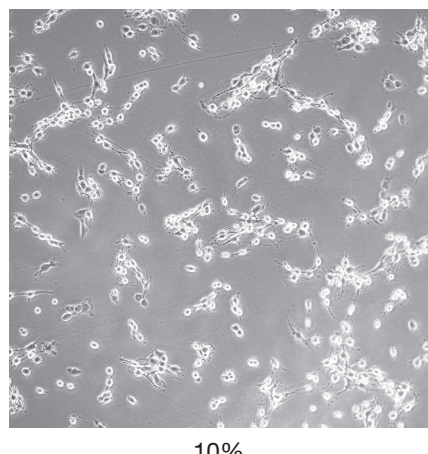

$10 \%$

Figure 1 Cell morphological changes after CSE intervention. As the concentration increases, skeletal muscle cells lose their typical shuttleshaped. They become rounded, the refraction increases, and cell floatation also increases $(\times 100)$. CSE, cigarette smoke extract.

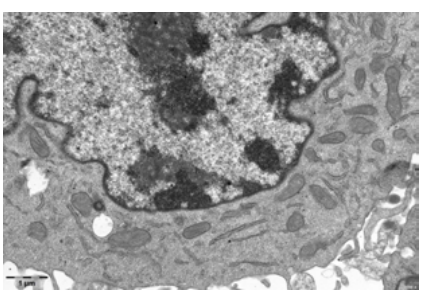

Control

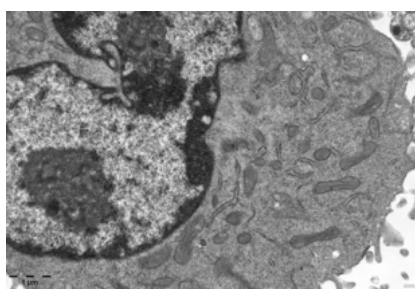

$2.5 \%$

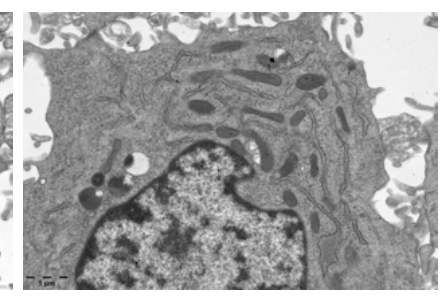

$5 \%$

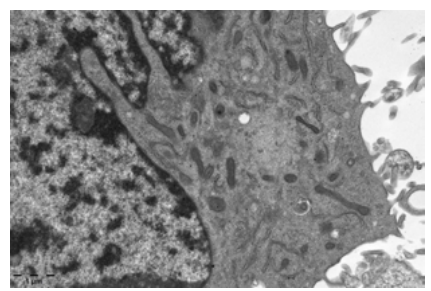

$10 \%$

Figure 2 The effect of CSE on mitochondria was observed by electron microscopy. As the concentration of CSE rises, the number of mitochondria increases, but the shape is mostly short and the mitochondrial cristae decrease. CSE, cigarette smoke extract.

concentration, the cells in the CSE intervention groups showed significantly reduced numbers. The cells became round, the gaps increased, the intercellular connection decreased, and the refraction increased. The cells floated, cell debris increased, and the arrangement order of regular outward extension was lost. Transmission electron microscopy showed that the number of mitochondria in CSE stimulated cells increased, but most of the length became shorter, like short rods. CSE stimulation reduced the mitochondrial crest of cells, and some of them showed vacuolated changes. The above changes were also CSE concentration dependent (Figures 1,2).

\section{Mitochondrial changes in cells}

Mitochondrial green fluorescence staining showed that with the increase of CSE concentration, intracellular green fluorescence decreased (Figure 3). The average green fluorescence values of the control group, $2.5 \%, 5 \%$, and $10 \%$ CSE intervention groups were $120.314 \pm 2.152$, $83.541 \pm 1.900,64.806 \pm 0.117$, and 56.450 1.360 , respectively. Decreased mitochondrial membrane potential is a hallmark event in the early stage of apoptosis, which can be detected by the conversion of red fluorescence to green fluorescence by JC-1 staining. Under inverted fluorescence microscopy of JC-1 staining, controls cells showed bright red fluorescence, while the green fluorescence was weak. The higher the concentration of CSE, the more obvious the green fluorescence (Figure 4). The mean red fluorescence values of the control group, $2.5 \%, 5 \%$, and $10 \%$ CSE intervention groups were $206.242 \pm 2.331,178.792 \pm 3.900$, $147.639 \pm 2.201$, and $119.470 \pm 4.580$, respectively. The mean green fluorescence values of the control group, $2.5 \%, 5 \%$, and $10 \%$ CSE intervention groups were $67.282 \pm 1.277$, $89.458 \pm 3.280,109.726 \pm 0.997$, and $157.812 \pm 1.129$, respectively. The red-green fluorescence ratios (\%) of the control group, $2.5 \%, 5 \%$, and $10 \%$ CSE intervention groups were $3.067 \pm 0.093,2.003 \pm 0.117,1.346 \pm 0.032$, and $0.758 \pm 0.035$, respectively. The above differences were statistically significant $(\mathrm{P}<0.05)$.

\section{Changes in Drp-1 mRNA expression}

The results of RT-PCR showed that with increased CSE 
A

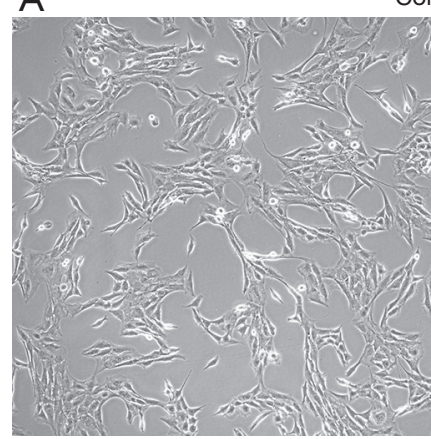

C

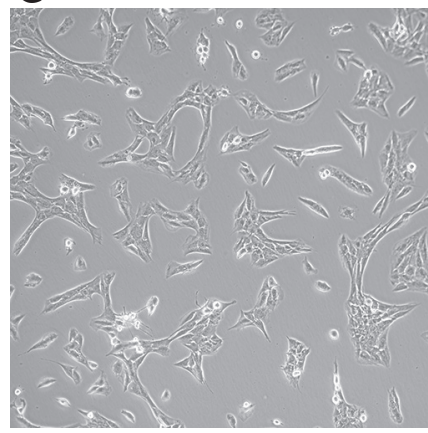

Control

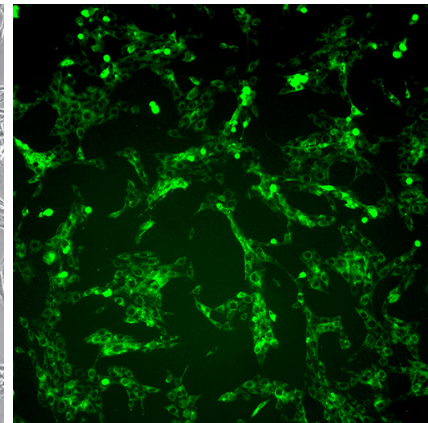

$5 \%$

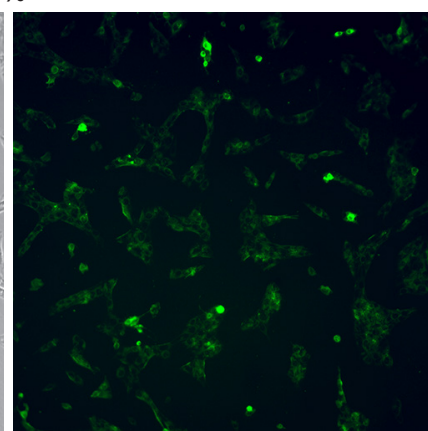

B
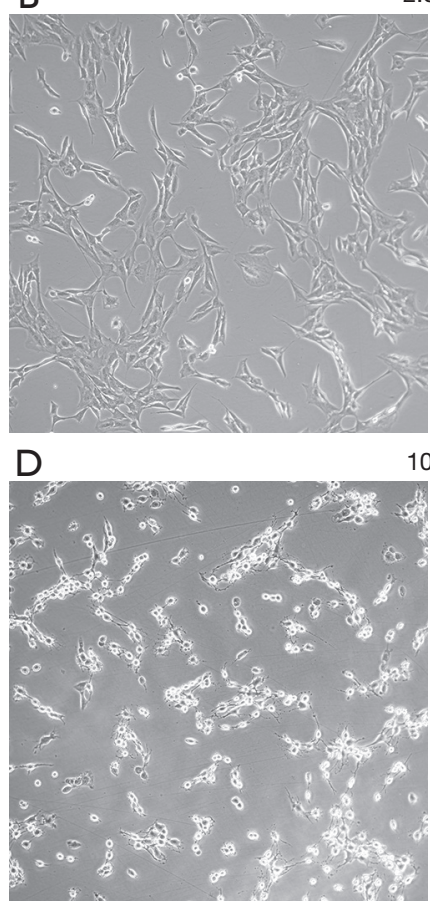

$2.5 \%$

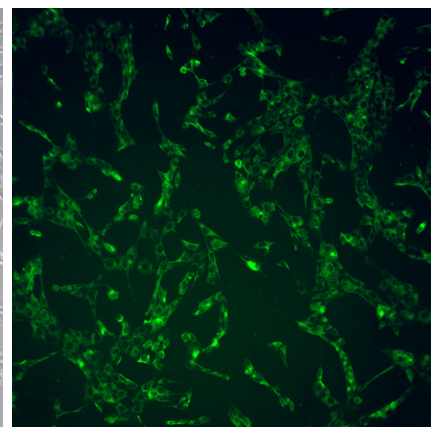

$10 \%$

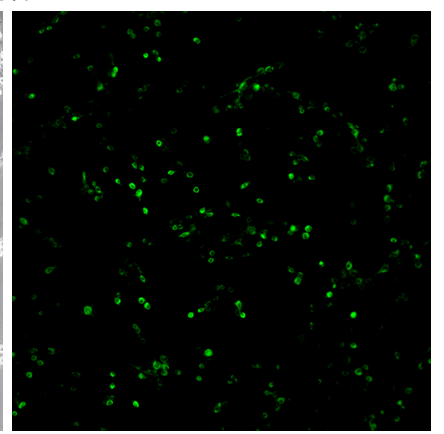

Figure 3 Mito-Tracker Green staining. It is a live-cell mitochondria-specific fluorescent staining. Green fluorescence decreased with increasing CSE concentration, suggesting a reduction in viable mitochondria $(\times 100)$. CSE, cigarette smoke extract.

concentration, the Drp-1 mRNA relative expression level increased, and the difference was statistically significant $(\mathrm{P}<0.05$, Figure 5).

\section{Altered Drp-1 protein expression}

Western blot results suggested that the higher the concentration of CSE, the higher the Drp-1 expression, and the difference was statistically significant $(\mathrm{P}<0.05$, Figure 6).

\section{Changes in cellular ROS}

Compared with the control group, flow cytometry suggested that cellular ROS were significantly increased after CSE intervention and increased with the concentration, and the difference was statistically significant $(\mathrm{P}<0.05$, Figure 7$)$.

\section{Changes in the apoptosis rate}

Compared with the control group, flow cytometry suggested that the apoptosis rates were significantly increased in the groups treated with CSE, and the difference was statistically significant $(\mathrm{P}<0.05$, Figure 8$)$.

\section{Discussion}

COPD is a common and frequently occurring disease in the respiratory department. Its main clinical manifestations are persistent respiratory symptoms and airflow limitation. The latest research shows that about $40 \%$ of patients have no serious impairment of pulmonary function but have significant SMD, and the mechanism is not clearly defined.

Mitochondria play an indispensable role in cellular energy production, redox regulation, and intracellular to extracellular calcium regulation, participating in not only cell differentiation and cell information transmission but also apoptosis and other processes. They also regulate cell growth and the cell cycle. Therefore, the ratio of mitochondrial division and fusion affects the normal physiological functions of cells. The dysfunction of mitochondria leads to reduced ATP, increased ROS, and improper calcium handling, which directly causes respiratory dysfunction and lung diseases such as idiopathic pulmonary fibrosis, asthma, and COPD (5-7). Studies have found that when muscle atrophy occurs, myocyte mitochondrial division is enhanced, and severe mitochondrial respiratory dysfunction develops, resulting 

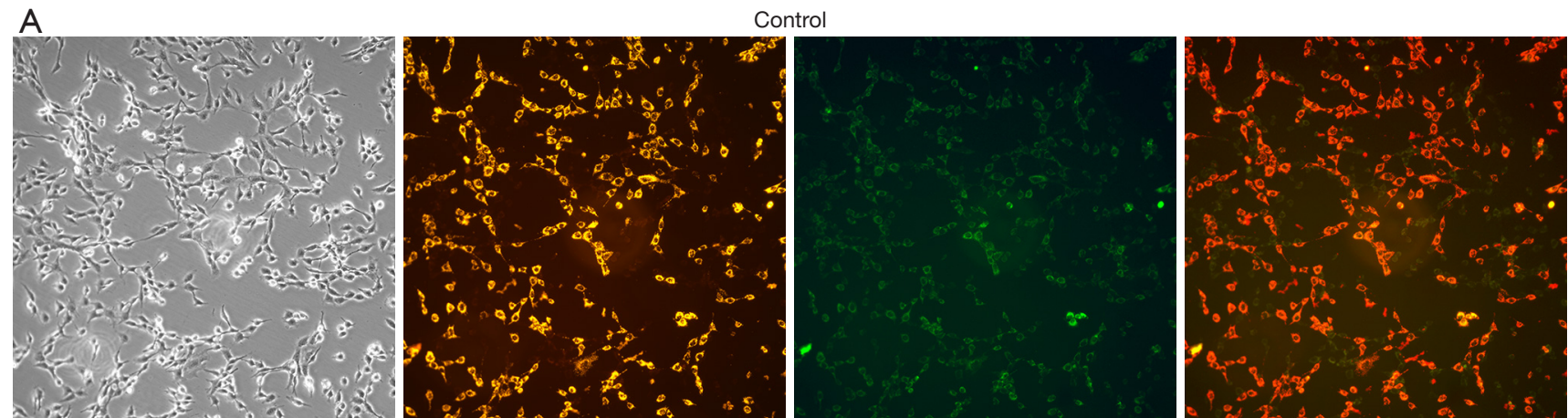

B
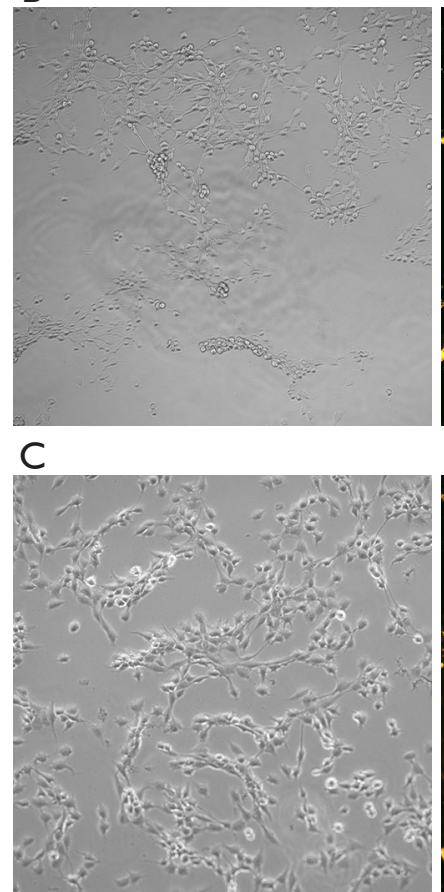

D
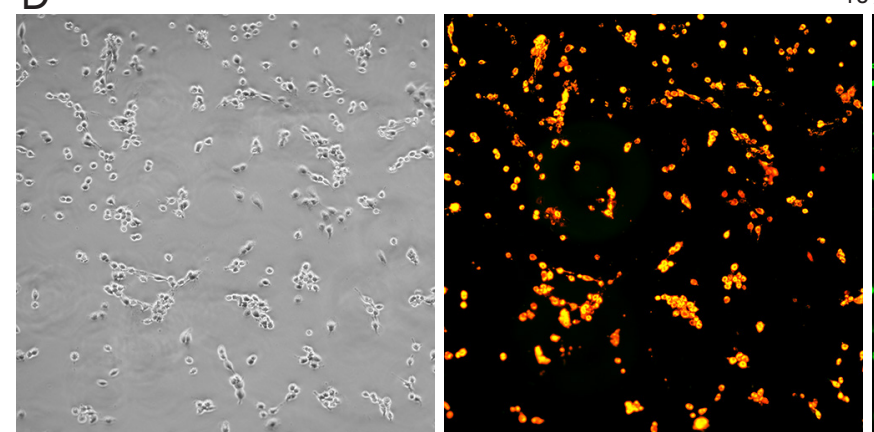

$10 \%$

Figure $4 \mathrm{JC}-1$ staining. As the concentration of CSE increased, the red/green fluorescence ratio increased, suggesting a decrease in

mitochondrial membrane potential) $(\times 100)$. CSE, cigarette smoke extract.
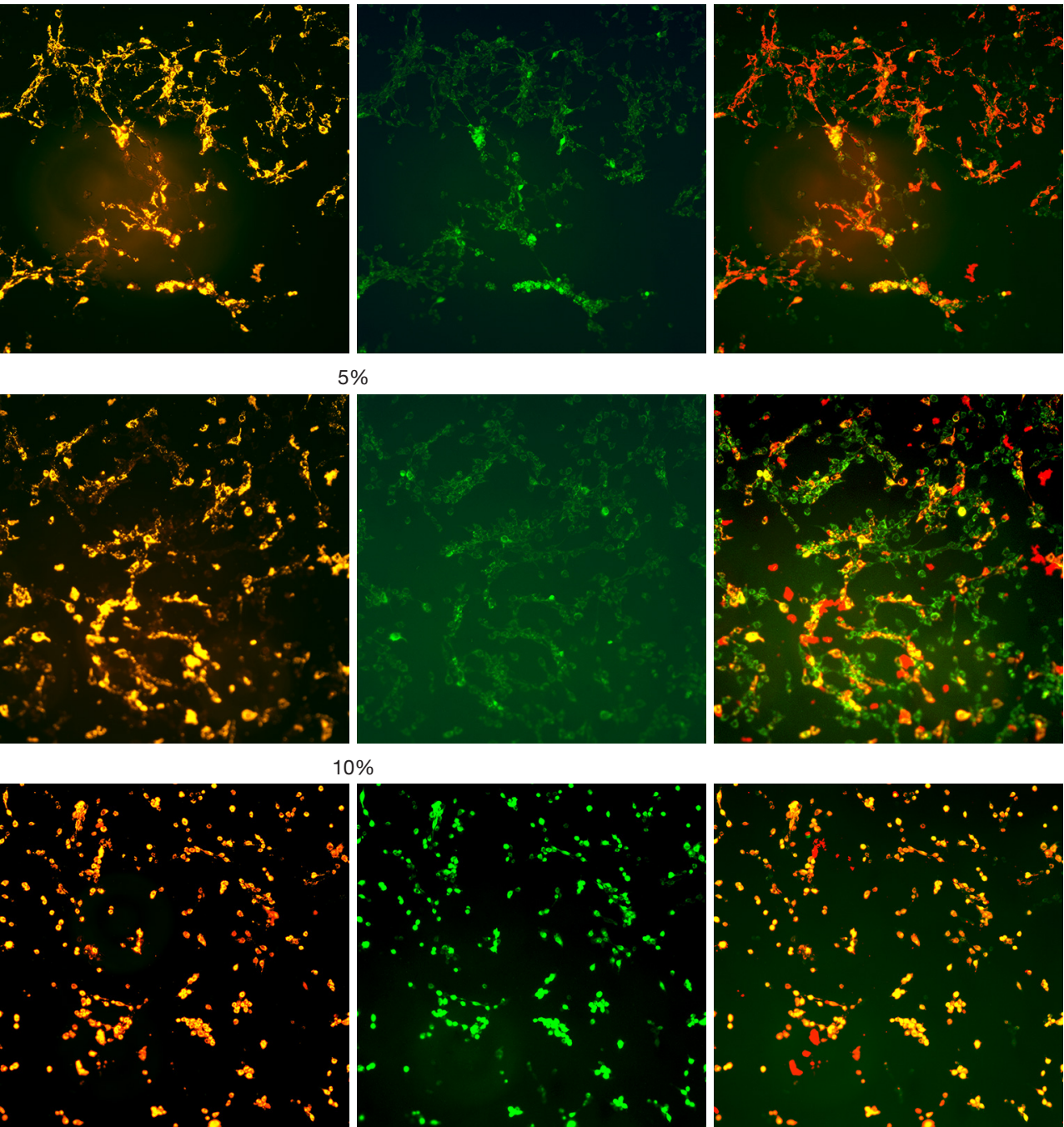
in decreased intracellular ATP. This in turn activates the AMP-dependent protein kinase (AMPK) signaling pathway, causing myocyte apoptosis, finally leading to the occurrence of muscle atrophy (8). There is increasing evidence that mitochondrial and ER calcium buffering and homeostasis

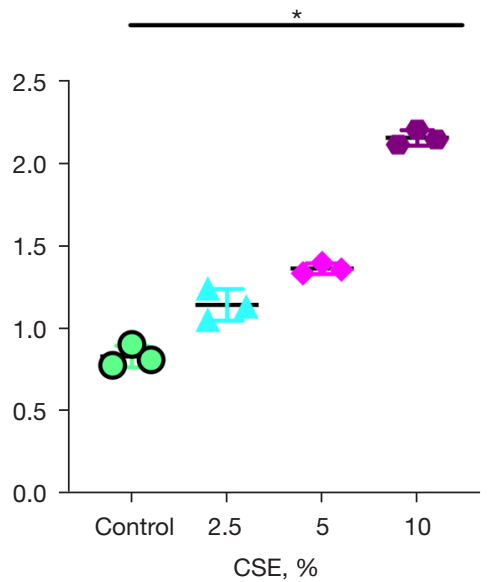

Figure 5 Changes in Drp-1 mRNA expression. The expression of Drp-1 RNA was Significant increased by CSE stimulation. ${ }^{*} \mathrm{P}<0.05$. Drp-1, Dynamin related protein 1; CSE, cigarette smoke extract. play an important role in cell proliferation, while cigarette smoke can lead to mitochondrial dysfunction and affect the buffering capacity of calcium $(9,10)$. Increases in intracellular and mitochondrial calcium will enhance the division of mitochondria (11).

Drp-1 is a key protein that initiates mitochondrial division and is mainly located in the cell matrix. When mitochondrial division occurs, Drp-1 is transferred from the cytoplasm to the division site of the outer mitochondrial membrane so that the mitochondria gradually compress

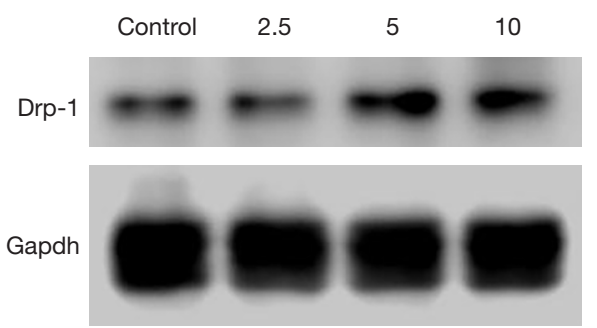

Figure 6 Changes in Drp-1 expression. Western blot showed that the expression of Drp-1 increased with increasing CSE concentration. Drp-1, Dynamin related protein 1; CSE, cigarette smoke extract.
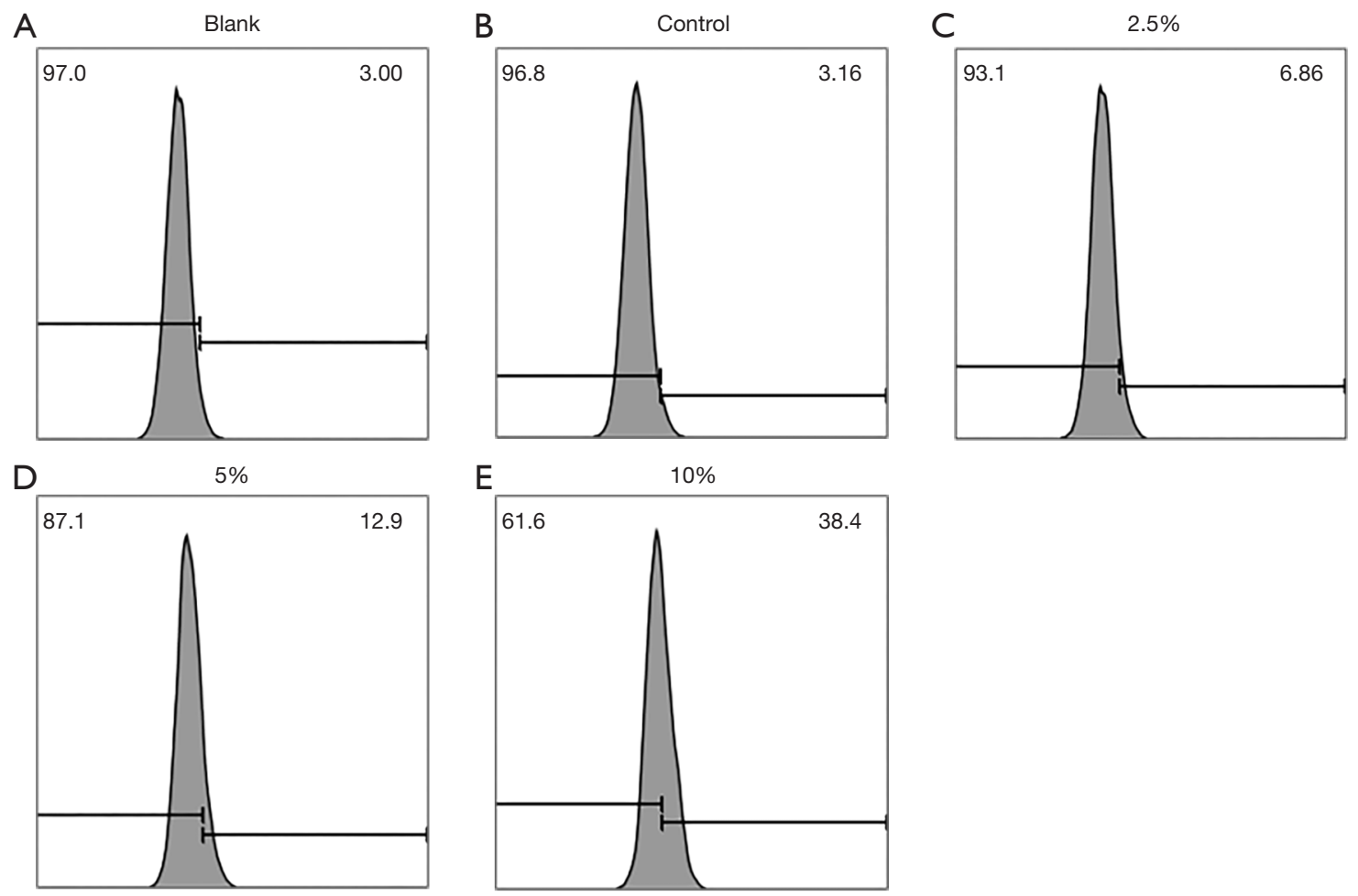

Figure 7 Changes in ROS. Compared with Control, ROS increased in $2.5 \%$ and $5 \%$, especially in $10 \%$. ROS, reactive oxygen species. 

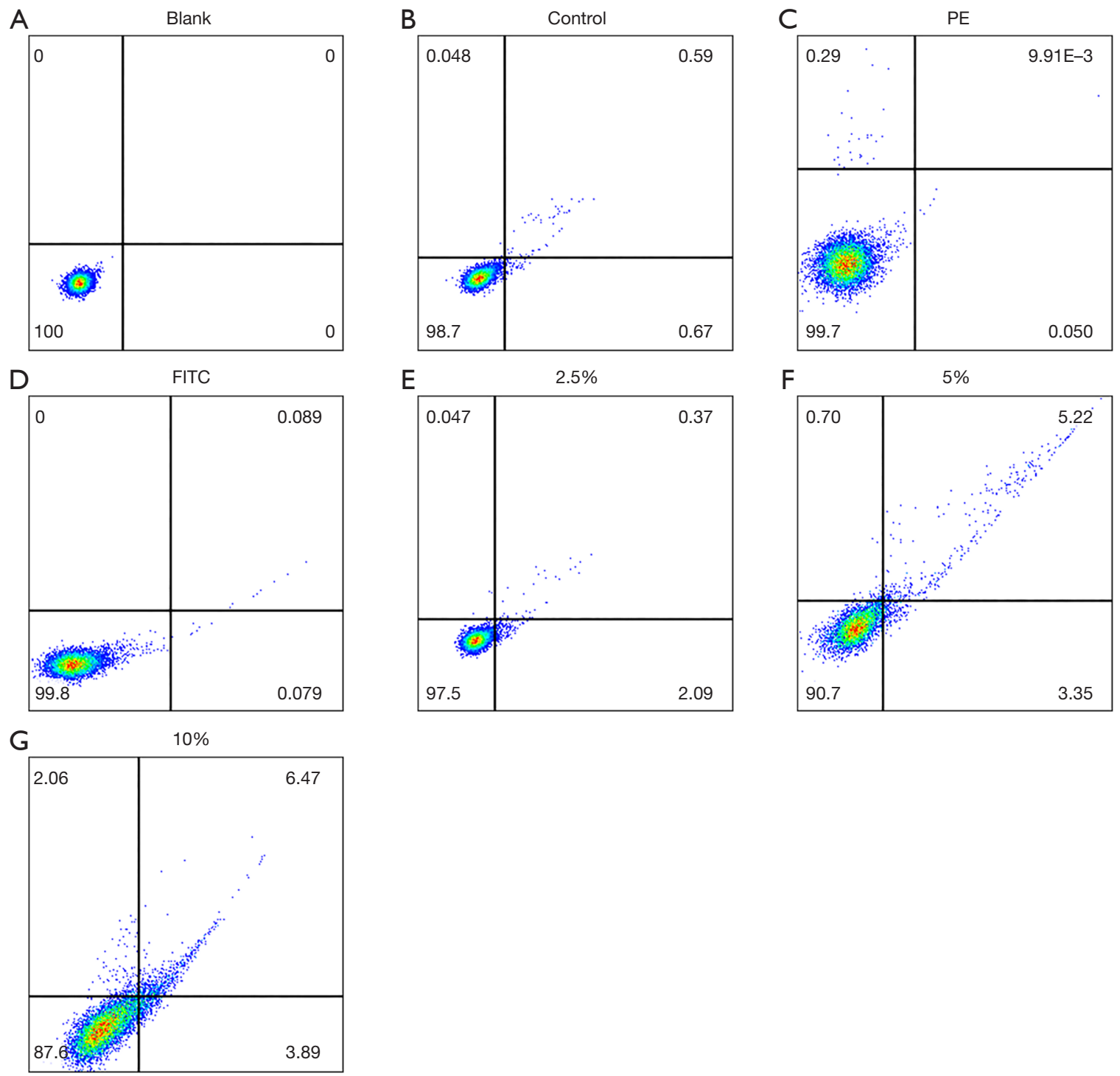

Figure 8 Changes in the apoptosis rate (CSE stimulation increased the rate of apoptosis of skeletal muscle cells compared with control. The higher the concentration, the more apoptosis). CSE, cigarette smoke extract.

until they break and mitochondrial division occurs. After the end of division, Drp-1 on the outer membrane returns to the cytoplasm, and the reciprocating movement of Drp-1 ensures the normal progress of mitochondrial division (12). Overexpression of Drp-1 inevitably enhances mitochondrial division. ROS are one-electron reduction products of a class of oxygen in the body, regulating several aspects of cell biology, including contraction, proliferation, and migration. Although ROS are indispensable in blocking physiological processes such as cell signaling and autophagy, overproduction of ROS is deleterious and can lead to cell damage and affects the contraction of myocytes (13).
Cellular damage by ROS is mainly characterized by damage to cellular DNA, carbohydrates, proteins, and lipids, which increases inflammatory responses $(14,15)$. Along with an increase in oxidative stress, ROS can also damage mitochondria, especially in COPD patients with mitochondrial dysfunction driving inflammation (16). As well as patients with COPD, mice exposed to oxidative stress have an increased probability of developing pulmonary inflammation and airway hyperresponsiveness, which are associated with decreased mitochondrial membrane potential and increased mitochondrial ROS generation. All these studies clearly demonstrate the role of mitochondria 
in the regulation of cellular oxidative stress and ROS generation. Consistent with other cells, mitochondria are also one of the major sites of ROS production in skeletal muscle cells. Therefore, the enhancement of mitochondrial division will undoubtedly lead to excessive production of ROS, which leads to increased cell damage or apoptosis.

In our study, mouse $\mathrm{C} 2 \mathrm{C} 12$ cells were used to observe the effects of different concentrations of CSE on cell biological behaviors, Drp-1 mRNA and protein expression, and mitochondrial division. We found that the cell numbers were significantly reduced. Compared with control cells, the cell groups stimulated with high concentrations of CSE $(5 \%, 10 \%)$ became round and floated, and cell debris increased. Western blot suggested that Drp-1 expression was significantly increased in skeletal muscle cells stimulated with high concentrations of CSE $(\mathrm{P}<0.05)$, which was consistent with the RT-PCR results. The above experimental results suggest that high concentrations of CSE stimulate the increased production of mitochondrial division proteins. Both Mito-Tracker Green and JC-1 are dyes for mitochondria in living cells. Mito-Tracker Green staining showed that green fluorescence decreased in the high concentration CSE groups, and the mean green fluorescence value decreased significantly in the $5 \%$ and $10 \%$ CSE groups compared with the $2.5 \%$ CSE group or the control group $(\mathrm{P}<0.05)$, indicating that high concentration CSE stimulation resulted in an increased level. JC-1 staining showed that control cells had bright red fluorescence and weak green fluorescence. The greater the CSE concentration, the more obvious the green fluorescence. There was a statistically significant difference in the mean red-green fluorescence ratio between the $5 \%$ and $10 \%$ CSE to $2.5 \%$ CSE groups or the control group $(\mathrm{P}<0.05)$. The shift of red fluorescence to green fluorescence in JC-1 staining suggests a decrease in mitochondrial membrane potential, which is a hallmark event in the early stages of apoptosis. Therefore, the JC-1 staining results in this study suggest that high concentrations of CSE lead to an increase in cell death or apoptosis.

In summary, CSE may increase the division of mitochondria by up-regulating the expression of Drp-1 in skeletal muscle, which leads to increased production of ROS, apoptosis, and ultimately skeletal muscle atrophy. Therefore, it can be speculated that smoking cessation or avoidance of a smoking environment may be helpful for the treatment of skeletal muscle atrophy in patients with COPD.

\section{Acknowledgments}

Funding: This work was supported by grant from Guangzhou science and technology plan projects (grant numbers: 2018A0303130342), Guangdong science and technology plan projects (grant numbers: 2017A070701014).

\section{Footnote}

Reporting Checklist: The authors have completed the MDAR reporting checklist. Available at https://dx.doi. org/10.21037/atm-21-5891

Data Sharing Statement: Available at https://dx.doi. org/10.21037/atm-21-5891

Conflicts of Interest: All authors have completed the ICMJE uniform disclosure form (available at https://dx.doi. org/10.21037/atm-21-5891). The authors have no conflicts of interest to declare.

Ethical Statement: The authors are accountable for all aspects of the work in ensuring that questions related to the accuracy or integrity of any part of the work are appropriately investigated and resolved.

Open Access Statement: This is an Open Access article distributed in accordance with the Creative Commons Attribution-NonCommercial-NoDerivs 4.0 International License (CC BY-NC-ND 4.0), which permits the noncommercial replication and distribution of the article with the strict proviso that no changes or edits are made and the original work is properly cited (including links to both the formal publication through the relevant DOI and the license). See: https://creativecommons.org/licenses/by-nc-nd/4.0/.

\section{References}

1. Chan SMH, Selemidis S, Bozinovski S, et al. Pathobiological mechanisms underlying metabolic syndrome (MetS) in chronic obstructive pulmonary disease (COPD): clinical significance and therapeutic strategies. Pharmacol Ther 2019;198:160-88.

2. Nishikawa H, Enomoto H, Ishii A, et al. Elevated serum myostatin level is associated with worse survival in patients with liver cirrhosis. J Cachexia Sarcopenia Muscle 2017;8:915-25. 


\section{Page 10 of 10}

3. Kong X, Yao T, Zhou P, et al. Brown Adipose Tissue Controls Skeletal Muscle Function via the Secretion of Myostatin. Cell Metab 2018;28:631-643.e3.

4. Jin CE, Zhen GH, Xu YJ, et al. Cigarette smoke extract and lipopolysaccharide reduce the expression of aquaporin 5 in mouse lung epithelial cells. Chinese Journal of Microcirculation 2010;20:29-31.

5. Wiegman CH, Michaeloudes C, Haji G, et al. Oxidative stress-induced mitochondrial dysfunction drives inflammation and airway smooth muscle remodeling in patients with chronic obstructive pulmonary disease. J Allergy Clin Immunol 2015;136:769-80.

6. Reddy PH. Mitochondrial Dysfunction and Oxidative Stress in Asthma: Implications for Mitochondria-Targeted Antioxidant Therapeutics. Pharmaceuticals (Basel) 2011;4:429-56.

7. Mabalirajan U, Ghosh B. Mitochondrial dysfunction in metabolic syndrome and asthma. J Allergy (Cairo) 2013;2013:340476.

8. Giacomello M, Pyakurel A, Glytsou C, et al. The cell biology of mitochondrial membrane dynamics. Nat Rev Mol Cell Biol 2020;21:204-24.

9. Delmotte P, Yang B, Thompson MA, et al. Inflammation alters regional mitochondrial $\mathrm{Ca}^{2}+$ in human airway smooth muscle cells. Am J Physiol Cell Physiol

Cite this article as: Tan Z, Li S, Zhu S, Yao X, Li J, Gao X, Yang S. Effect of cigarette smoke extract on mitochondrial division in mouse quadriceps femoris cells. Ann Transl Med 2021;9(22):1699. doi: 10.21037/atm-21-5891

\section{Tan et al. CSE enhance mitochondrial division in quadriceps cells}

2012;303:C244-56.

10. Aravamudan B, Kiel A, Freeman M, et al. Cigarette smokeinduced mitochondrial fragmentation and dysfunction in human airway smooth muscle. Am J Physiol Lung Cell Mol Physiol 2014;306:L840-54.

11. Bravo-Sagua R, Parra V, López-Crisosto C, et al. Calcium Transport and Signaling in Mitochondria. Compr Physiol 2017;7:623-34.

12. Lessard SJ, MacDonald TL, Pathak P, et al. JNK regulates muscle remodeling via myostatin/SMAD inhibition. Nat Commun 2018;9:3030.

13. Olszewski MA, Robinson NE, Yu MF, et al. Effects of hydrogen peroxide on isolated trachealis muscle of horses. Am J Vet Res 1995;56:1479-85.

14. Henricks PA, Nijkamp FP. Reactive oxygen species as mediators in asthma. Pulm Pharmacol Ther 2001;14:409-20.

15. Suski JM, Lebiedzinska M, Bonora M, et al. Relation between mitochondrial membrane potential and ROS formation. Methods Mol Biol 2012;810:183-205.

16. Wiegman CH, Li F, Ryffel B, et al. Oxidative Stress in Ozone-Induced Chronic Lung Inflammation and Emphysema: A Facet of Chronic Obstructive Pulmonary Disease. Front Immunol 2020;11:1957. 